\title{
TOXIC DISORDERS
}

\section{PRENATAL PCB EXPOSURE AND COGNITIVE DYSFUNCTION}

The neuropsychological effects of in utero exposure to PCBs and their related compounds were evaluated in 27 'Yu-Cheng' ('oil disease') children (ages 7 to 12 years) at the Departments of Pediatrics and Psychiatry, National Cheng Kung University, Tainan, Taiwan. Full-scale IQ scores on the WISC-R were significantly lower than in the 27 controls. Mean P300 latencies of auditory event-related potentials were significantly longer and the amplitudes reduced. Pattern visual evoked potentials and somatosensory evoked potentials were unaffected. Apart from a slight increase in soft signs, the neurologic examinations of the exposed children were not different from controls. (Chen Y-J, Hsu C-C. Effects of prenatal exposure to PCBs on the neurological function of children: A neuropsychological and neurophysiological study. Dev Med \& Child Neurol April 1994; $\underline{36}: 312-320$ ). (Respond: Dr Yung-Jung Chen, Dept of Pediatrics, Medical College, National Cheng Kung University, 138 Sheng-Li Road, Tainan 70428, Taiwan, R.O.C.).

COMMENT. PCBs affect cognitive function of children exposed in utero. Evoked potentials are useful in examining the neurotoxicity of environmental pollutants in the young child. The P300 wave latency is related to the solving of cognitive tasks and the amplitude of P300 reflects concentration abilities. A significantly greater difference in P300 latencies was found for exposed children with lower IQ scores compared to controls. Delayed effects of PCBs on newborns whose mothers consumed contaminated Lake Michigan fish during pregnancy have been reported; smaller head circumference and growth retardation persisted beyond infancy and short-term memory and behavioral deficits occurred at later follow-up (see Ped Neur Briefs Jan 1990, and March 1993).

\section{FETAL VALPROATE SYNDROME IN SIBLINGS}

Clinical and neurodevelopmental findings in four children (two sibling pairs) exposed in utero to valproic acid are reported from the Departments of Paediatrics and Human Genetics, University of Witaterstrand, Johannesburg, South Africa. Three were globally developmentally delayed with marked speech disability, characteristic dysmorphic features, and one with autism. The fourth child had dysmorphism and a learning disability. (Christianson AL et al. Fetal valproate syndrome: Clinical and neurodevelopmental features in two sibling pairs. Dev Med \& Child Neurol April 1994; $\underline{36}: 357-369)$. (Respond: Dr Arnold L Christianson, Dept of Human Genetics and Developmental Biology, University of Pretoria Faculty of Medicine, PO Box 2034, Pretoria 0001, South Africa).

COMMENT. Future children could be at increased risk of fetal valproate syndrome if valproate is continued during pregnancy. The drug itself, the dosage, the genetic susceptibility, and other environmental factors are involved in the etiology of drug-related birth defects. In addition to adverse effects on the fetus, valproate is reported to cause reproductive disorders, including polycystic ovaries and hyperandrogenism, in women with epilepsy (see Ped Neur Briefs Dec 1993). 